\title{
MINIREVIEW
}

\section{Evolution underground: shedding light on the diversification of subterranean insects}

\author{
Carlos Juan*1 and Brent C Emerson ${ }^{2}$ \\ See research article http://www.biomedcentral.com/1471-2148/10/29
}

\begin{abstract}
A recent study in $B M C$ Evolutionary Biology has reconstructed the molecular phylogeny of a large Mediterranean cave-dwelling beetle clade, revealing an ancient origin and strong geographic structuring. It seems likely that diversification of this clade in the Oligocene was seeded by an ancestor already adapted to subterranean life.
\end{abstract}

Cave organisms have long been considered a model system for testing evolutionary and biogeographic hypotheses because of their isolation, simplicity of community structure and specialization. Adaptation to cave environments promotes the regression of functionless (unused) characters across a broad taxonomic range, in concert with evolutionary change in other morphological traits. Change typically involves the degeneration of eyes and loss of pigments, while at the same time appendices become elongated, intensification of sensory organs occurs, and life cycles become modified -a syndrome known as troglomorphy [1].

Modes of speciation and explanations for the geographic distributions of subterranean animals have both been debated (see for example [2,3]). Extrinsic environmental factors causing extinction of surface ancestors pre-adapted to the subterranean (such as glaciations or aridification) are suggested to be a mechanism forcing populations underground. This model is usually invoked in limestone cave systems of continental temperate regions. However, among tropical faunas, in which surface (epigean) taxa are often found co-occurring with subterranean sister taxa, active colonization of the subterranean habitat is suggested as a more plausible

\footnotetext{
*Correspondence: cjuan@uib.es

'Department de Biologia, Universitat de les IIles Balears, 07122 Palma de Mallorca, Spain

Full list of author information is available at the end of the article
}

explanation [2]. Mirroring this debate, both the development of a topographic or ecological barrier resulting in the separation of a once continuously distributed ancestral population or species into separate populations (vicariance) and dispersal, have been discussed as contrasting factors shaping subterranean animal distributions. Vicariance is typically considered the dominant of these two processes, as subterranean species have very limited dispersal potential, particularly in ecologically unsuitable areas [4].

Testing hypotheses of origin and adaptation among subterranean taxa has been hindered by the inherent difficulties of sampling the rare and more elusive cave taxa and extensive morphological convergence caused by strong selection pressures imposed by the subterranean environment [4]. In recent years molecular phylogenies have been obtained for numerous taxonomic groups containing subterranean lineages, permitting rigorous comparisons of competing evolutionary hypotheses. In a study published in BMC Evolutionary Biology, Ribera et al. [5] have investigated the origin and evolution of a diverse lineage of subterranean beetles of the tribe Leptodirini (family Leiodidae) (Figure 1a), focusing on the distribution of this group in the western Mediterranean. This study is one of the first in which the evolutionary history of a presumably monophyletic group composed of mostly subterranean species is examined using molecular data. Samples of a large number of species from genera occurring in the Iberian Peninsula plus representatives from Sardinia and the Carpathians are included in the study. DNA sequences totalling 4 kilobases from five mitochondrial and two nuclear DNA fragments were used to construct robust phylogenies using different methods and to quantify diversification patterns and times from molecular clock calibrations.

\section{Speciation in cave-dwelling organisms}

The evolutionary passage from the surface to a terrestrial or aquatic subterranean environment involves an array of adaptations to a highly specialized habitat characterized by permanent darkness and peculiar ecological features, such as humidity in terrestrial caves, scarce and patchy 


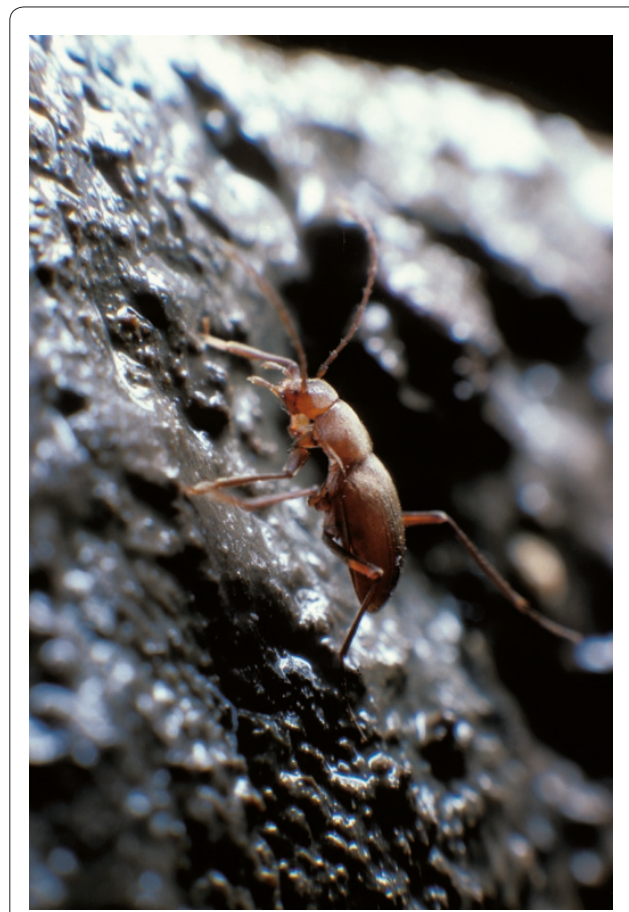

(a)

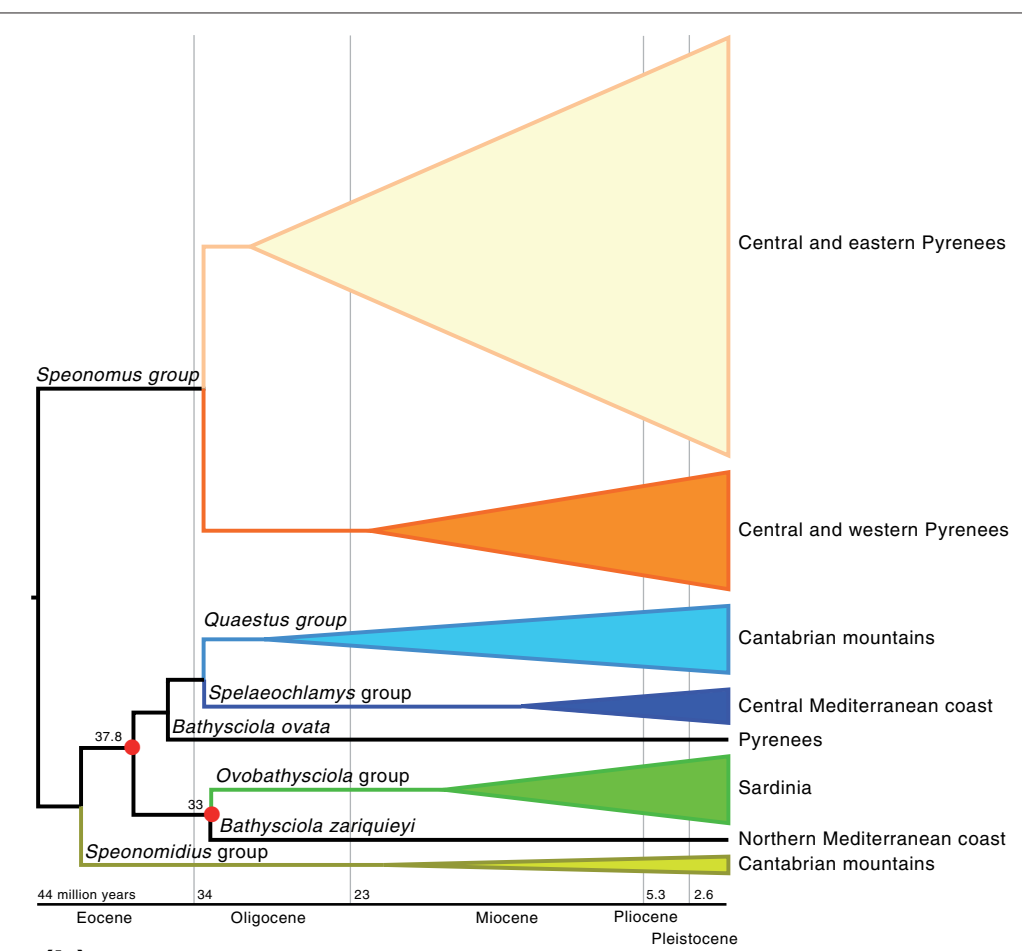

(b)

Figure 1. Cave-beetles and phylogenies. (a) Photograph of the cave-beetle species Cytodromus dapsoides (Leptoridini, Leiodidae) from the Vercors National Park in Southeast France. The tribe Leptodirini includes about 235 genera and around 900 species, most of them exclusively subterranean. The highest diversity is found in the north and east of the Iberian Peninsula, Corsica and Sardinia, the southern Alps, Balkan Peninsula, Romania and southern Russia, the Caucasus, Middle East and Iran. (b) Simplified phylogenetic tree obtained by Ribera et al. [5] using combined mitochondrial and nuclear sequences. The tree was linearized (fitted to constancy of molecular substitution rate) using Bayesian methods. Red circles indicate tree nodes used for calibration of the molecular clock using the mitochondrial gene cox 1 only (considering 33 million years ago for the age of initial separation of Sardinian species from their sister lineage), and including all mitochondrial sequence information but excluding species from Sardinia (from which only cox 1 sequences were available). In the latter case an estimated age of 37.8 million years ago was used for the separation of Bathysciola zariquieyi from its sister. The width of each clade is proportional to the number of species included in the study. The basal Speonomidius lineage includes the muscicolus genus Notidocharis. A geological timeline with the relevant epochs is provided below the tree. Figure 1a courtesy of Christian Vanderbergh.

food resources, and constant temperature. Many evolutionary studies of subterranean taxa have concentrated on the origin of the lineages and the tempo and mode of troglomorphic adaptations [3].

The geographic distributions and phylogenetic relationships of surface taxa and related subterranean taxa can help to distinguish between the two key hypotheses of origin; the parapatric (termed in the specialized literature the 'adaptive shift hypothesis') and allopatric (the 'climatic relict hypothesis') models (Figure 2; [2,3,6] and references therein). Parapatric describes the situation where two species or distinct populations of the same species overlap to a limited extent, while allopatric describes the situation where species or populations have mutually exclusive distributions.

The allopatric model predicts that surface species either became extinct as a result of climatic change, leaving only cave-adapted populations remaining, or survive in geographic areas that were unaffected by climatic change (and are thus allopatric with regard to the subterranean populations). Within this model an extrinsic cessation of gene flow caused by extirpation of the surface populations explains the origin of cavedwelling organisms. In contrast, the adaptive shift hypothesis advocates differentiation by divergent natural selection and consequent reduction of gene flow between surface and underground populations. The prediction from this is that closely related epigean and subterranean species will have parapatric distributions [2]. However, reduced power to distinguish between these hypotheses, or even incorrect inference, can be expected if extinction of only some epigean populations or species has occurred, or if there have been significant changes in geographic distributions [3]. 


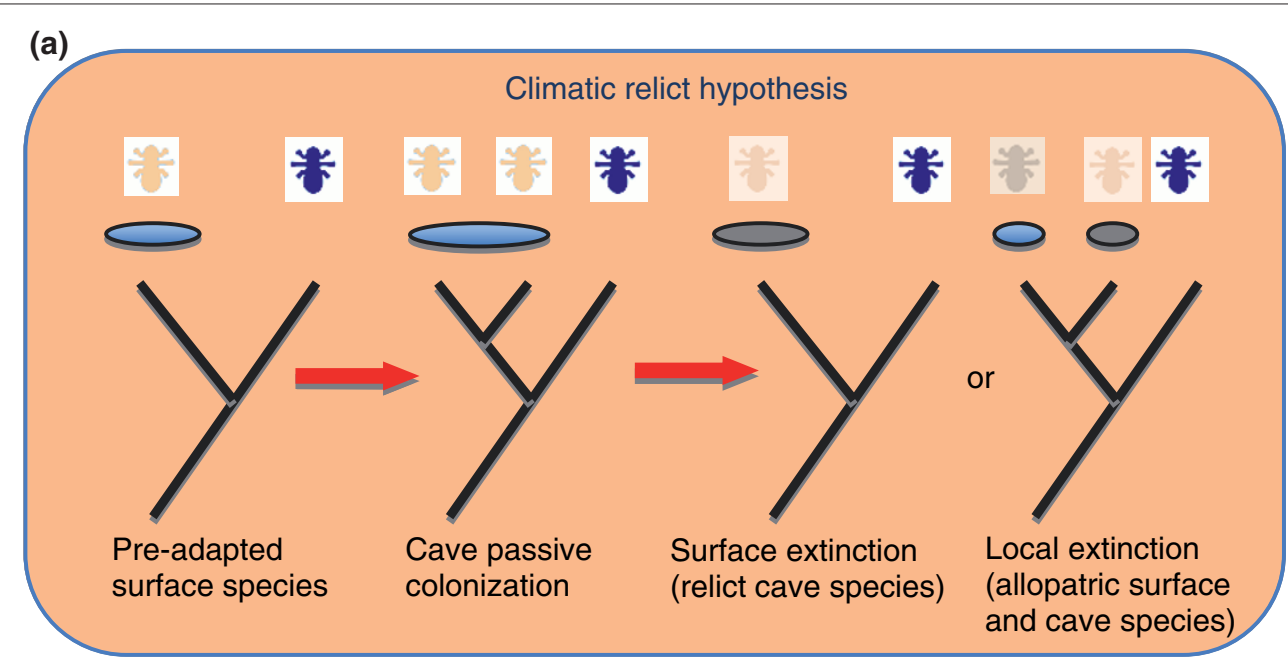

\section{(b)}

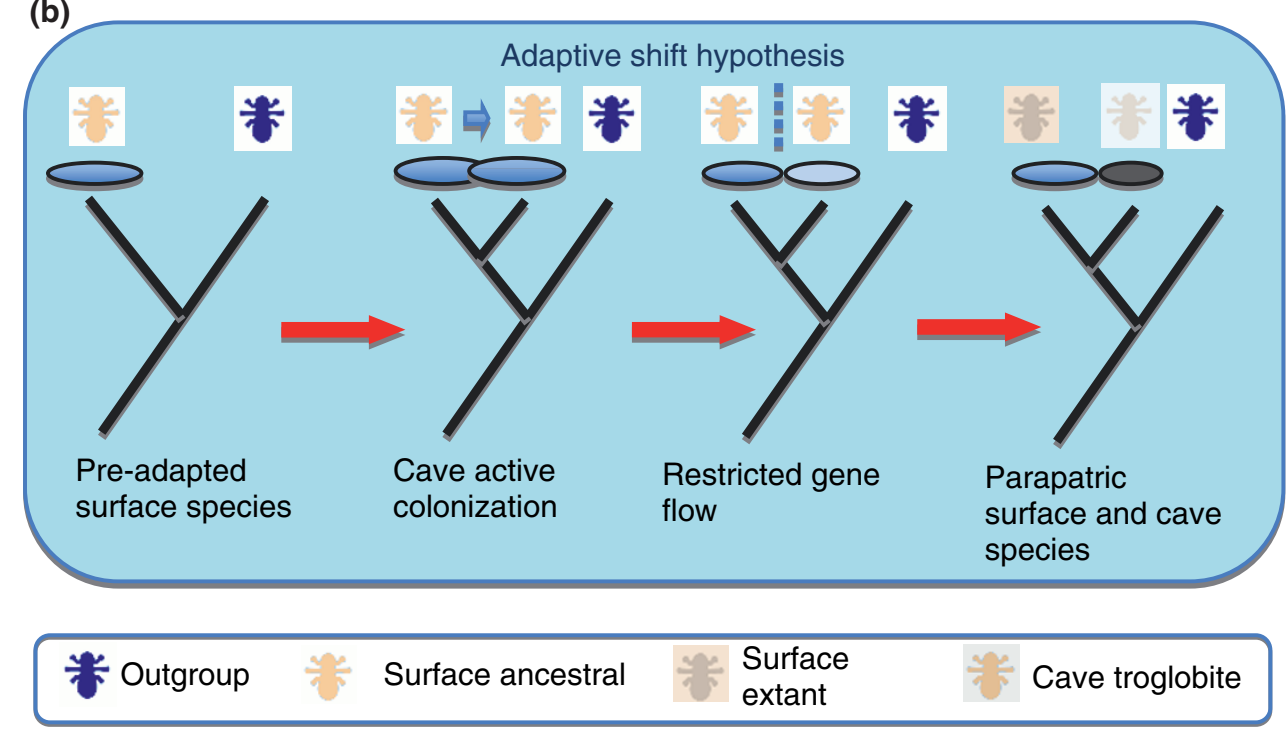

Figure 2. Speciation models in subterranean taxa. Schematic diagram of (a) the 'climatic relict' and (b) the'adaptive shift' hypotheses. In the former, a broadly distributed surface species that has exaptations (pre-adaptations) to the underground environment invades the caves. The underground population remains in contact with the surface population, limiting genetic divergence of the two. Climatic oscillations cause local extinction of surface populations, whereas surviving populations remain in the underground. The predictions from this for geographic distribution are that either only relict cave-dwelling lineages survive, or surface populations are strictly allopatric and geographically remote with respect to the underground. Over time, cave populations differentiate, developing troglomorphic characters and become reciprocally monophyletic. In the adaptive shift hypothesis caves are invaded by surface populations, exploiting new resources with the establishment of differential selection pressures in the epigean and underground environments. Speciation is driven by divergent selection accompanied by a reduction of gene flow. In this case, surface and cave species are expected to have parapatric distributions, at least during the initial phases of the process. Ellipses represent geographic distributions of populations. Troglobite is the term given to animals that have become adapted to dwell in cave environments and that cannot survive outside such environments. Diagram modified from Figures 1 and 2 in [2].

\section{Inferring patterns of subterranean evolution from molecular phylogenies}

Reconstruction of evolutionary surface-to-subterranean transitions in a range of arthropods (for example, terrestrial and aquatic beetles, crickets, spiders, amphipods, isopods, shrimps and crayfish) and vertebrates (for example, fish and salamanders) deduced from molecular phylogenies has lent support to both the parapatric and allopatric models, with parapatric distributions being more frequently recovered in tropical regions (for example, in Hawaiian isopods and Canary Island beetles and spiders) [7]. This could be due to the lack of climatic events affecting surface populations, to geologic factors, or because of recent adaptation to cave life in these areas. In some of these cases geographically restricted lineages include subterranean and surface species, showing 
evidence of multiple colonization of the subterranean habitat [8].

The study of Ribera et al. [5] focuses on a monophyletic beetle lineage that is composed primarily of subterranean taxa, with only one lineage representing species with eyes, living in moss habitats (genus Notidocharis). Although the genus Notidocharis has been assumed to be the sister-group of the remaining Leptoridini, Ribera et al. find that it occupies an ambiguous position close to the origin of the clade [5]. This basal placement of Notidocharis and the fact that some subterranean lineages have not been sampled limits definitive conclusions about the relationship of subterranean species with surface relatives. However, given the monophyly of Notidocharis, the number of subterranean lineages, and the geographic distribution of subterranean clades, the pattern strongly suggests that extensive speciation has occurred within the underground domain.

The major monophyletic subterranean Leptoridini lineages are geographically structured in the mountain massifs of the Iberian Peninsula and the deduced associated divergence times reveal ancient divisions (Figure 1b). The conclusion, given the absence of surface relatives, is that diversification took place within the subterranean habitat from ancestors adapted to cave life via dispersal and subsequent isolation due to geographical factors causing vicariance [5]. It is unclear which of these latter two factors has prevailed, but this conclusion does imply greater dispersal ability than has been previously recognized for subterranean animals.

\section{Molecular clocks and estimation of the age of subterranean lineages}

The correlation of divergence times of surface-tosubterranean transitions with independent estimates of paleoclimatic or geologic events can be used to explain extinction of ancestors or the origin of new subterranean habitats [3]. 'Molecular clock' approaches, based on the quasi-constant rate of molecular evolution, have been extensively used in evolutionary biology to estimate the age of origin of taxa. However, the scarcity of fossil data for many taxonomic groups requires the use of extrinsic nucleotide substitution rates obtained from other organisms and/or genes, or calibration of the clock from biogeographic datings of vicariant species or populations.

The time of separation of the Sardinian microplate from the continent has previously been used to estimate rates of nucleotide change in the gene for mitochondrial cytochrome oxidase subunit 1 ( $\operatorname{cox} 1)$ in subterranean Leptodirini [1]. That study concluded that Sardinian and Pyrenean species diversification occurred during the mid to late Miocene (16 to 4.6 million years ago), contemporaneously with ecological and geologic changes in the western Mediterranean region. In the more comprehensive study of Ribera et al. [5], the authors date the origin of the main Pyrenean Leptodirini clades to be approximately 34 million years ago, close to the formation of the Pyrenees and the beginning of its cave formation (early Oligocene), whereas the Cantabrian massif of the Iberian Peninsula and coastal Mediterranean clades appear to be even older [5] (Figure 1b).

\section{Challenges and future directions}

Explaining subterranean insect origin can prove to be more complicated than distinguishing between simple allopatric or parapatric models, and may require careful estimation of ongoing or historical gene flow between the two habitats [6]. For instance, a repeated colonization of lava tubes with local extinction of some of the surface populations due to progressive drought has been suggested to explain the origin of subterranean amphipods in the island of La Palma in the Canary archipelago [8]. It has also been pointed out that important parameters such as dispersal capacity, population size, and ability to adapt and persist have been probably underestimated in subterranean animals [9]. In addition, a considerable number of previously unrecognized (morphologically cryptic) species are increasingly being revealed by molecular data, especially in animals that live in subterranean groundwater (stygobionts), in some cases contradicting what have appeared to be widespread distributions [10,11]. The use of conserved molecular markers should prove particularly exciting for future studies of extreme disjunct biogeographic distributions in stygobiont crustaceans [12], and the devising of appropriate models to understand post-colonization speciation within the caves on the basis of individual case studies [7].

\section{Acknowledgements}

We are grateful to Christian Vanderbergh for providing the photograph in Figure 1a. CJ's research on subterranean crustaceans is supported by Spanish grants CGL2006-01365 and CGL2009-08256, and his research visit to the University of East Anglia (Norwich) has been funded by the Ministerio de Educación (Spain), project PR2009-0231.

\section{Author details}

'Department de Biologia, Universitat de les Illes Balears, 07122 Palma de Mallorca, Spain

${ }^{2}$ Centre for Ecology, Evolution and Conservation, School of Biological Sciences, University of East Anglia, Norwich NR4 7TJ, UK

\section{Published: 11 March 2010}

\section{References}

1. Caccone A, Sbordoni V: Molecular biogeography of cave life: a study using mitochondrial DNA from bathysciine beetles. Evolution 2001, 55:122-30.

2. Rivera MA, Howarth FG, Taiti S, Roderick GK: Evolution in Hawaiian caveadapted isopods (Oniscidea: Philosciidae): vicariant speciation or adaptive shifts? Mol Phylogenet Evol 2002, 25:1-9.

3. Leys R, Watts C, Cooper S, Humphreys W: Evolution of subterranean diving beetles (Coleoptera: Dytiscidae: Hydroporini, Bidessini) in the arid zone of Australia. Evolution 2003, 57:2819-2834.

4. Porter M: Subterranean biogeography: what have we learned from 
molecular techniques? J Cave Karst Stud 2007, 69:179-186.

5. Ribera I, Fresneda J, Bucur R, Izquierdo A, Vogler AP, Salgado JM, Cieslak A: Ancient origin of a Western Mediterranean radiation of subterranean beetles. BMC Evol Biol 2010, 10:29.

6. Niemiller M, Fitzpatrick B, Miller B: Recent divergence with gene flow in Tennessee cave salamanders (Plethodontidae: Gyrinophilus) inferred from gene genealogies. Mol Ecol 2008, 17:2258-2275.

7. Guzik M, Cooper S, Humphreys W, Austin A: Fine-scale comparative phylogeography of a sympatric sister species triplet of subterranean diving beetles from a single calcrete aquifer in Western Australia. Mol EcOl 2009, 18:3683-3698

8. Villacorta C, Jaume D, Oromí P, Juan C: Under the volcano: phylogeography and evolution of the cave-dwelling Palmorchestia hypogaea (Amphipoda, Crustacea) at La Palma (Canary Islands). BMC Biol 2008, 6:7.

9. Buhay J, Crandall K: Subterranean phylogeography of freshwater crayfishes shows extensive gene flow and surprisingly large population sizes. $\mathrm{Mol}$
Ecol 2005, 14:4259-4273.

10. Lefebure T, Douady CJ, Malard F, Gibert J: Testing dispersal and cryptic diversity in a widely distributed groundwater amphipod (Niphargus rhenorhodanensis). Mol Phylogenet Evol 2007, 42:676-686.

11. Zakšek V, Sket B, Gottstein S, Franjević D, Trontelj P: The limits of cryptic diversity in groundwater: phylogeography of the cave shrimp Troglocaris anophthalmus (Crustacea: Decapoda: Atyidae). Mol Ecol 2009, In press.

12. Page T, Humphreys W, Hughes J: Shrimps down under: evolutionary relationships of subterranean crustaceans from Western Australia (Decapoda: Atyidae: Stygiocaris). PLoS ONE 2008, 3:e1618.

\section{doi:10.1186/jbiol227}

Cite this article as: Juan C, Emerson BC: Evolution underground: shedding light on the diversification of subterranean insects. Journal of Biology 2010, 9:17. 